Pacific Journal of Mathematics

QUANTIFIERS AND ORTHOMODULAR LATTICES 


\title{
QUANTIFIERS AND ORTHOMODULAR LATTICES
}

\author{
M. F. JANOWITZ
}

1. Introduction. The "logic" of (non-relativistic) quantum mechanics is currently thought of as being the lattice of closed subspaces of a separable infinite dimensional Hilbert space [7, p. 49]. It has been speculated by P. Jordan [5] that this "logic" ought not to be a lattice at all, but rather what he calls a skew lattice. Given a lattice $L(\cap, \cup)$, Jordan observes that if one has functions $f, F: L \rightarrow L$ satisfying the conditions

$$
\begin{aligned}
(a f \cup b) f & =a f \cup b f \\
a f & \leqq a \\
(a \cap b F) F & =a F \cap b F \\
a & \leqq a F ;
\end{aligned}
$$

then $L(\wedge, \vee)$ is a skew lattice, where the operations $\wedge, \vee$ are defined by:

$$
\begin{aligned}
& a \wedge b=a \cap b F \\
& a \vee b=a f \cup b .
\end{aligned}
$$

Skew lattices themselves will not concern us here; rather we shall be interested in mappings on lattices having the above properties. Such mappings turn out to be gereralizations of universal and existential quantifiers. With this thought in mind it seems of interest to begin an investigation of quantifiers on an orthomodular lattice, and in particular to consider the lattice $L(H)$ of closed subspaces of a Hilbert space $H$, determining all mappings $f, F$ defined on $L(H)$ and satisfying Jordan's prescription.

The remaining part of this section will be devoted to a brief outline of known definitions and theorems that will prove useful in the sequel. These results can essentially be found in [1], [2], [9] and [10] but are included here for convenience. An orthomodular lattice is a lattice $L$ with 0 and 1 equipped with an orthocomplementation ': $L \rightarrow L$ and which satisfies the orthomodular identity $e \leqq f \Rightarrow f=e \vee\left(f \wedge e^{\prime}\right)$. Henceforth $L$ will always represent an orthomodular lattice. If $e, f \in L$ with $e \leqq f$ it is easily shown that the interval $L(e, f)=$ $\{g \in L: e \leqq g \leqq f\}$ is itself an orthomodular lattice with orthocomplementation

$$
g \rightarrow g^{\sharp}=e \vee\left(f \wedge g^{\prime}\right)=\left(e \vee g^{\prime}\right) \wedge f .
$$

Received May 10, 1962. The results in this paper are part of the author's doctoral dissertation (Wayne State University, 1963) written under the direction of Professor D. J. Foulis. 
The semigroup (under function composition) of all monotone maps $\varphi: L \rightarrow L$ is denoted by $M(L)$. Two monotone maps $\varphi$ and $\psi$ are said to be mutually adjoint in case $(e \varphi)^{\prime} \psi \leqq e^{\prime}$ and $(e \psi)^{\prime} \varphi \leqq e^{\prime}$ for all $e \in L$. If $\varphi \in M(L)$ has an adjoint then this adjoint is unique [1, p. 651], and is denoted by $\varphi^{*}$. The subset of $M(L)$ possessing adjoints in $M(L)$ is denoted by $S(L)$. It is shown in [1] that $S(L)$ is a Baer *-semigroup (under function composition) and that every $\varphi \in S(L)$ is a hemimorphism of $L$; i.e., that $0 \varphi=0$ and $(e \vee f) \varphi=e \varphi \vee f \varphi$ for every $e, f \in L$. In fact every $\varphi \in S(L)$ preserves arbitrary suprema whenever they exist in $L$.

There corresponds to each $e \in L$ a mapping $\varphi_{e}: L \rightarrow L$ given by $f \rightarrow f \varphi_{e}=\left(f \vee e^{\prime}\right) \wedge e$, where $\varphi_{e}=\varphi_{e}^{2}=\varphi_{e}^{*} \in S(L), f \varphi_{e}=f$ if and only if $f \leqq e$, and $f \varphi_{e}=0$ if and only if $f \leqq e^{\prime}$ (see [1, p. 652] and [10, pp. 300-301]). The element $f$ is said to commute with $e$ (written $f C e$ ) in case $f \varphi_{e}=f \wedge e$. For future reference we state the next. results in the form of a lemma.

Lemma 1. Let $e, f, g \in L$. Then:

(i) $f \leqq e \Rightarrow f C e$.

(ii) $f C e \Rightarrow e C f$.

(iii) $f C e \Rightarrow f C e^{\prime}$.

(iv) If any two of the three conditions eCf, $f C g$, eCg hold, then $(e \vee f) \wedge g=(e \wedge g) \vee(f \wedge g)$ and $(e \wedge f) \vee g=(e \vee g) \wedge(f \vee g)$.

(v) If $f C e_{\alpha}$ for each $\alpha \in A$, and if $\mathrm{V}_{\alpha \in A} e_{\alpha}$ exists then $f C \mathrm{~V}_{\alpha \in A} e_{\alpha}$.

Proof. See [2] and [9].

In [11, p. 240] J. von Neumann defines the center of a continuous geometry. This notion can be carried over to an abitrary orthomodular lattice by decreeing that the center of $L$, in symbols $C(L)$, is the set of all those elements of $L$ which commute with every other element. It then follows [2] that $0,1 \in C(L)$, and $C(L)$ is a Boolean sublattice closed under the formation of orthocomplements and of arbitrary suprema and infima whenever they exist in $L$.

The lattice $L$ is said to be irreducible in case 0 and 1 are the only elements with unique complements. It can easily be shown that $e \in C(L)$ if and only if $e$ has a unique complement, from which it follows that $L$ is irreducible if and only if $C(L)=\{0,1\}$.

Before proceeding the author would like to thank Professor D. J. Foulis for the many helpful suggestions he has made during the writing of this paper.

2. Quantifiers. Generalizing a notion of Halmos [3, p. 220] a mapping $\varphi: L \rightarrow L$ will be called a quantifier on $L$ in case $\varphi$ satisfies: 
(Q1) $0 \varphi=0$.

(Q2) $e \leqq e \varphi$ for all $e \in L$.

(Q3) $(e \wedge f \varphi) \varphi=e \varphi \wedge f \varphi$ for all $e, f \in L$.

It should be noted that there are always two special quantifiers on $L$ :

(i) The discrete quantifier = identity map;

(ii) The indiscrete (or as in Halmos [3] simple) quantifier $e \varphi=1$ for $e \neq 0,0 \varphi=0$. We now proceed to investigate some of the properties of quantifiers and will find all quantifiers on an atomic orthomodular lattice whose center is complete as a sublattice.

THEOREM 2. Let $\varphi$ be a quantifier on L. Then:

(i) $1 \varphi=1$.

(ii) $\varphi=\varphi^{2}$.

(iii) $e \leqq f \Rightarrow e \varphi \leqq f \varphi$.

(iv) $(e \varphi)^{\prime} \varphi=(e \varphi)^{\prime}$.

(v) $\varphi$ is a projection in $S(L)$.

(vi) $(L) \varphi=$ the set of fixed points of $\varphi$ is a sublattice of $L$ closed with respect to the formation of orthocomplements and of arbitrary suprema and infima whenever they exist in $L$.

Proof. (i) $1 \leqq 1 \varphi$, so $1=1 \varphi$.

(ii) $e \varphi^{2}=(e \varphi) \varphi=(1 \wedge e \varphi) \varphi=1 \varphi \wedge e \varphi=e \varphi$.

(iii) If $e \leqq f$, then $e \leqq f \varphi, e=e \wedge f \varphi$, $e \varphi=(e \wedge f \varphi) \varphi=e \varphi \wedge f \varphi$, so that $e \varphi \leqq f \varphi$.

(iv) Note that $0=0 \varphi=\left[(e \varphi)^{\prime} \wedge(e \varphi)\right] \varphi=(e \varphi)^{\prime} \varphi \wedge e \varphi$. By the orthomodular identity, $(e \varphi)^{\prime} \leqq(e \varphi)^{\prime} \varphi$ implies that $(e \varphi)^{\prime} \varphi=$ $(e \varphi)^{\prime} \vee\left[(e \varphi)^{\prime} \varphi \wedge e \varphi\right]=(e \varphi)^{\prime}$.

(v) This follows immediately from (ii), (iii) and the observation that $(e \varphi)^{\prime} \varphi=(e \varphi)^{\prime} \leqq e^{\prime}$.

(vi) If $e \varphi=e$ then $e^{\prime} \varphi=(e \varphi)^{\prime} \varphi=(e \varphi)^{\prime}=e^{\prime}$. The result is now clear in view of the fact that $\varphi=\varphi^{2} \in S(L)$ and the known properties of $S(L)$.

LEMMA 3. Let $\varphi$ be a quantifier on $L, a$ an atom in $L, e=a \varphi$. Then:

(i) $\left.\varphi\right|_{L(0) e}$ is indiscrete.

(ii) $e$ is an atom in $(L) \varphi$.

(iii) For any $f \in L$, either $e \wedge f=0$ or $e \leqq f \varphi$.

Proof. (i ) Let $b \in L(0, e)$, so $b \varphi \leqq e \varphi=e$. Since $a$ is an atom, $a \wedge b \varphi=a$ or 0 . But if $a \wedge b \varphi=a, e=a \varphi=(a \wedge b \varphi) \varphi=a \varphi \wedge b \varphi=$ $e \wedge b \varphi=b \varphi$; while $a \wedge b \varphi=0$ implies that $0=0 \varphi=(a \wedge b \varphi) \varphi=$ $e \wedge b \varphi=b \varphi$, whence $b=0$.

(ii) If $b \in(L) \varphi$ and $b \leqq e$, then $b=b \varphi=0$ or $e$. 
(iii) Suppose $e \wedge f \neq 0$. Then $(e \wedge f) \varphi=e \wedge f \varphi \neq 0$, and by (ii), $e \leqq f \varphi$.

We borrow another idea of Halmos [3] and call a mapping $\varphi: L \rightarrow L$ a symmetric closure operator in case $\varphi$ is a hemimorphism, $\varphi=\varphi^{2}$, and for each $e \in L, e \leqq e \varphi$ and $(e \varphi)^{\prime} \varphi \leqq e^{\prime}$. It is shown by Halmos [3, Th. 3] that in a Boolean lattice the notions of quantifier and symmetric closure operator coincide. It is interesting to observe that in an orthomodular lattice this result does not quite carry over. By Theorem 2, every quantifier is a symmetric closure operator. One can show without difficulty that every center valued symmetric closure operator is a quantifier; however, if $a \notin C(L)$, the mapping $\alpha_{\alpha}: L \rightarrow L$ defined by $e \alpha_{\alpha}=(e \vee a) \wedge\left(e \vee \alpha^{\prime}\right)$ is an example of a symmetric closure operator that is not a quantifier. It follows from this that $L$ is a Boolean lattice if and only if every symmetric closure operator is a quantifier.

One can define the central cover of $e$, in symbols $e \nu$, to be the infimum of the set of central elements $z$ such that $e \leqq z$, provided of course that such an infimum exists. Note that if ev exists for all $e \in L$, then $\nu$ is a non-trivial example of a quantifier. Clearly $0 \nu=0$, $e \leqq e \nu$ for every $e \in L$, and $e=e \nu$ if and only if $e \in C(L)$. We also have that if $e \leqq f$, then $e \leqq f \nu \in C(L)$, whence $e \nu \leqq f \nu$. It then follows that $e \nu^{2}=(e \nu) \nu=e \nu$, and $(e \nu)^{\prime} \nu=(e \nu)^{\prime} \leqq e^{\prime}$ so that $\nu=\nu^{2}=\nu^{*} \in S(L)$. But then $\nu$ is a center valued symmetric closure operator, hence a quantifier.

Lemma 4. Suppose that ev exists for each $e \in L$. Then $\varphi$ is a center valued quantifier on $L$ if and only if $\varphi=\nu \circ \alpha$, where $\alpha$ is $a$ quantifier on $C(L)$. The decomposition is unique in that $\alpha=\left.\varphi\right|_{o(L)}$.

Proof. Evidently $\nu \circ \alpha$ is always a center valued quantifier. If conversely $\varphi$ is a center valued quantifier, set $\alpha=\left.\varphi\right|_{o(L)}$. Then $e \leqq e \nu \leqq e \varphi$, whence $e \varphi \leqq e \nu \varphi=e \nu \alpha \leqq e \varphi^{2}=e \varphi$ for all $e \in L$, from which it follows that $\varphi=\nu \circ \alpha$. The uniqueness of the decomposition is obvious.

Lemma 5. Let $\varphi$ be a quantifier on $L, a$ any atom of $L$. Then $a \varphi \wedge f \neq 0 \Rightarrow a \varphi C f$.

Proof. If $a=a \varphi$ the result is obvious, so we can suppose $a<a \varphi$. Suppose that $a \varphi \wedge f \neq 0$ and $a \varphi C f$ fails. Routine computation shows that $k=a \varphi \varphi_{f}, \vee(a \varphi \wedge f)$ is a complement of $(a \varphi)^{\prime}, k \neq a \varphi$, and $k \wedge a \varphi \neq 0$. By Lemma 3, $a \varphi \leqq k \varphi$, and since $k \wedge(a \varphi)^{\prime}=0,\left(k \wedge(a \varphi)^{\prime}\right) \varphi=$ $k \varphi \wedge(a \varphi)^{\prime}=0$. Applying the orthomodular identity to $a \varphi \leqq k \varphi$, we 
have $k \varphi=a \varphi \vee\left(k \varphi \wedge(a \varphi)^{\prime}\right)=a \varphi$, whence $k \leqq a \varphi$. A second application of the orthomodular identity now produces $a \varphi=k \vee\left(a \varphi \wedge k^{\prime}\right)=k$, a contradiction. Hence $a \varphi \wedge f \neq 0 \Rightarrow a \varphi C f$.

LEMMA 6. If $\varphi$ is a quantifier on an atomic lattice $L$, then $a<a \varphi$ for an atom $a$ implies that a $\varphi$ is central.

Proof. If $b$ is any atom such that $b<b \varphi$, then $b^{\prime}<b^{\prime} \varphi=1$, so that if $a \varphi \wedge b^{\prime}=0$, we would have $\left(a \varphi \wedge b^{\prime}\right) \varphi=a \varphi \wedge b^{\prime} \varphi=a \varphi=0$, a contradiction. Hence $a \varphi \wedge b^{\prime} \neq 0$, and by Lemma 5 , $a \varphi C b$.

Consider next an atom $b=b \varphi$. Since $a<a \varphi$, we can write $a \varphi=$ $a \vee\left(a \varphi \wedge a^{\prime}\right)$. Now $a<a \varphi \Rightarrow a^{\prime} \varphi=1 \Rightarrow b \wedge a^{\prime} \neq 0 \Rightarrow b C a$. Similarly, $\left(a \varphi \wedge a^{\prime}\right)^{\prime} \varphi=\left[(\alpha \varphi)^{\prime} \vee a\right] \varphi=(a \varphi)^{\prime} \vee a \varphi=1$, so that $b C\left(a \varphi \wedge a^{\prime}\right)$. By Lemma 1, $a \varphi C b$. This shows that $a \varphi$ commutes with every atom. In an atomic orthomodular lattice each element is the supremum of the atoms it dominates, so by Lemma $1(v), a \varphi \in C(L)$.

CoRollary. Let $L$ be atomic and irreducible. Then $L$ admits only the discrete and the indiscrete quantifiers.

If $L=L_{1} \times L_{2}$, and $\varphi_{1}, \varphi_{2}$ are quantifiers on $L_{1}, L_{2}$, respectively, it is easily shown that $\varphi=\varphi_{1} \times \varphi_{2}$ is a quantifier on $L$. It can also be shown that an element $a$ is central in $L_{1}$ if and only if $(a, 0)$ is central in $L$. Let $h \in C(L)$, and let us adopt the notation $\nu_{h}=\left.\nu\right|_{L(0, h)}$, $\alpha_{h}=$ a quantifier on $C(L(0, h))=C(L) \cap L(0, h), I_{h}=$ the identity map restricted to $L(0, h)$. We then have:

THEOREM 7. Let $L$ be atomic with $C(L)$ complete as a sublattice. Then $\varphi$ is a quantifier on $L$ if and only if $\varphi=\left(\nu_{h} \circ \alpha_{h}\right) \times I_{h^{\prime}}$ for some $h \in C(L)$.

Proof. Any mapping of the form $\left(\nu_{h} \circ \alpha_{h}\right) \times I_{h^{\prime}}$ is evidently a quantifier on $L$. If conversely $\varphi$ is a quantifier on $L$, set $h=$ $\sup \left\{a_{\nu} \varphi: a_{\nu}\right.$ is an atom, $\left.a_{\nu} \varphi \in C(L)\right\}$, and write $L=L(0, h) \times L\left(0, h^{\prime}\right)$. If $b \leqq h$ is an atom, then since each $a_{\nu} \varphi$ is central, $b=b \wedge h=h \varphi_{b}=$ $\left(\mathrm{V}_{\nu} a_{\nu} \varphi\right) \varphi_{b}=\mathrm{V}_{\nu}\left(a_{\nu} \varphi \varphi_{b}\right)=\mathrm{V}_{\nu}\left(a_{\nu} \varphi \wedge b\right)$. Thua for some index $\mu, b \leqq a_{\mu} \varphi$; by Lemma $3, b \varphi=a_{\mu} \varphi \in C(L)$. This shows that $\left.\varphi\right|_{L(0 h)}$ is center valued, and by Lemma 4 we may write $\left.\varphi\right|_{L(0 h)}=\nu_{h} \circ a_{h}$. By Lemma 6, $\left.\varphi\right|_{L\left(0 h^{\prime}\right)}=I_{h^{\prime}}$. Hence $\varphi=\left(\nu_{h} \circ \alpha_{h}\right) \times I_{h^{\prime}}$.

It should be observed that since Halmos [3] has essentially determined all quantifiers on a Boolean lattice, the above theorem enables us to find all quantifiers on an atomic orthomodular lattice with complete center. 
3. Atomic bisection and irreducibility. Generalizing a property of the lattice of closed subspaces of a Hilbert space, let us say that a pair $(b, c)$ of distinct atoms can be bisected in case there exists an atom $a \leqq b \vee c$ such that $a \neq b$ and $a \neq c$. The lattice $L$ has the atomic bisection property if $L$ is atomic and every pair of distinct atoms can be bisected. The reader will no doubt notice that the results of this section will go through under the weakened hypothesis that pairs of orthogonal atoms can be bisected. This, however, is only an apparent weakening since for non-orthogonal atoms $(b, c)$, we can write $b \vee c=b \vee\left[(b \vee c) \wedge b^{\prime}\right]$; any atom $a \leqq(b \vee c) \wedge b^{\prime}$ will then bisect $(b, c)$.

LEMMA 8. If $L$ has the atomic bisection property, then so does any interval $L(e, f)$.

Proof. The lemma follows immediately from the fact that the mapping $a \rightarrow a \wedge e^{\prime}$ is an orthocomplement preserving lattice isomorphism of $L(e, f)$ onto $L\left(0, f \wedge e^{\prime}\right)$.

LEMMA 9. If $L$ has the atomic bisection property, then $L$ is irreducible.

Proof. Suppose that $L$ is atomic, and $h \in C(L)$ with $h \neq 0, h \neq 1$. Choose atoms $b, c$ with $b \leqq h, c \leqq h^{\prime}$. Then for any atom $a \leqq b \vee c$, $a=a \wedge 1=a \wedge\left(h \vee h^{\prime}\right)=(a \wedge h) \vee\left(a \wedge h^{\prime}\right)$, so that $a \leqq h$ or $a \leqq h^{\prime}$. But $a \leqq h$ implies that $a \leqq(b \vee c) \wedge h=(b \wedge h) \vee(c \wedge h)=b$, and since $b$ is an atom, $a=b$. Similarly $a \leqq h^{\prime}$ implies $a=c$, so atomic bisection fails.

LEMMA 10. If $L$ is atomic complete and modular then irreducibility of $L$ is both a necessary and sufficient condition for $L$ to have the atomic bisection property.

Proof. By a theorem of I. Kaplansky [6] every complete modular orthocomplemented lattice is a continuous geometry. The result now follows immediately from [8, p. 80, Th. 2.4].

Combining the above results with the observation that if the pair $(b, c)$ of distinct atoms cannot be bisected, then $L(0, b \vee c)$ is the Boolean lattice $\{0, b, c, b \vee c\}$, we have the following theorem:

THEOREM 11. Let $L$ be atomic. Then the following conditions are mutually equivalent:

(i) L has the atomic bisection property.

(ii) Every interval $L(e, f)$ is irreducible. 
(iii) $L(0, b \vee c)$ is irreducible for all atoms $b, c$ in $L$. If in addition $L$ is complete and modular, we can add:

(iv) $L$ is irreducible.

4. Determination of the weak quantifiers on $L(H)$. By a weak quantifier on $L$ we will mean a mapping $\varphi: L \rightarrow L$ satisfying axioms (Q2) and (Q3) of $\S 2$. A glance at the proof of Theorem 2 shows that parts (i)-(iii) will carry over for weak quantifiers. It should be noted that the reason for our interest in weak quantifiers is that these (together with their duals) are precisely the functions needed for constructing a skew lattice by the method of Jordan.

THEOREM 12. Let $\varphi$ be a weak quantifier on $L$. Then:

(i) $\left.\varphi\right|_{L(0 \varphi 1)}$ is a quantifier.

(ii) $\varphi$ preserves arbitrary suprema whenever they exist in $L$.

Proof. (i) By hypothesis $\left.\varphi\right|_{L(0 \varphi \text { 1) }}$ satisfies axioms (Q2) and (Q3), so we need only note that $(0 \varphi) \varphi=0 \varphi$.

(ii) Let $e, f \in L$. Then $e, f \leqq e \vee f$, so that $e \varphi, f \varphi \leqq(e \vee f) \varphi$; hence $e \varphi \vee f \varphi \leqq(e \vee f) \varphi$. The reverse inequality follows from the fact that $e \vee f \leqq e \varphi \vee f \varphi$, so $(e \vee f) \varphi \leqq(e \varphi \vee f \varphi) \varphi=e \varphi \vee f \varphi$, since $\left.\phi\right|_{L(0 \varphi 1)}$ is a quantifier.

Suppose now that $e=\mathrm{V}_{\alpha} e_{\alpha}$ exists. Then $e \vee 0 \varphi=\left(\mathrm{V}_{\alpha} e_{\alpha}\right) \vee 0 \varphi=$ $\mathrm{V}_{\alpha}\left(e_{\alpha} \vee 0 \varphi\right)$, so

$$
\begin{aligned}
e \varphi & =e \varphi \vee 0 \varphi=(e \vee 0 \varphi) \varphi \\
& =\left[\mathrm{V}_{\alpha}\left(e_{\alpha} \vee 0 \varphi\right)\right] \varphi=\mathrm{V}_{\alpha}\left(e_{\alpha} \vee 0 \varphi\right) \varphi \\
& =\mathrm{V}_{\alpha}\left(e_{\alpha} \varphi \vee 0 \varphi\right)=\left(\mathrm{V}_{\alpha} e_{\alpha} \varphi\right) \vee 0 \varphi=\mathrm{V}_{\alpha} e_{\alpha} \varphi .
\end{aligned}
$$

It now seems natural to call $\varphi$ a discrete or an indiscrete weak quantifier according to whether $\left.\varphi\right|_{L(0 \varphi, 1)}$ is the discrete or the indiscrete quantifier on $L(0 \varphi, 1)$. Our immediate goal is to determine all weak quantifiers on a lattice having the atomic bisection property. From Theorem 11 and the Corollary to Lemma 6, we see that every weak quantifier on such a lattice is discrete or indiscrete. It suffices then to find all discrete and indiscrete weak quantifiers on an arbitrary orthomodular lattice $L$.

Our labor is greatly diminished by the observation that for any weak quantifier $\varphi$ on $L$, $e \varphi=(e \vee(0 \varphi)) \hat{\varphi}$ for each $e \in L$, where $\hat{\varphi}=$ $\left.\varphi\right|_{L(0 \varphi, 1)}$. It follows that if $\varphi$ is indiscrete, $e \varphi=0 \varphi$ for all $e \leqq 0 \varphi$, and $e \varphi=1$ otherwise. Conversely for any $a \in L$ the prescription $e \varphi=a$ for $e \leqq a, e \varphi=1$ for $e \leqq a$, defines an indiscrete weak quantifier, so this completely determines all indiscrete weak quantifiers on $L$. 
In case $\varphi$ is discrete it is immediate that $e \varphi=[e \vee(0 \varphi)] \hat{\rho}=$ $e \vee(0 \varphi)$. Our next task is to find necessary and sufficient conditions on $a$ which will assure us that the mapping $e \rightarrow e \vee a$ is a discrete weak quantifier. We will use the notation $M(b, c)$ to indicate that the pair $(b, c)$ is modular; i.e., that $a \leqq c \Rightarrow(a \vee b) \wedge c=a \vee(b \wedge c)$.

THEOREM 13. Given $a \in L$ the mapping $\psi_{a}$ defined $b y b \psi_{a}=b \vee a$ is a discrete weak quantifier if and only if $M\left(e, a^{\prime}\right)$ for all $e \in L$. It then follows that $L$ is modular if and only if $\psi_{a}$ is a discrete weak quantifier for every $a \in L$.

Proof. We clearly need only concern ourselves with axiom (Q3). Suppose first that $\psi_{a}$ is a weak quantifier. Then for any $e, f \in L$, $\left(e \wedge f \psi_{a}\right) \psi_{a}=e \psi_{a} \wedge f \psi_{a}$, so $[e \wedge(f \vee a)] \vee a=(e \vee a) \wedge(f \vee a)$. Now if $b \geqq a$ then $b=b \vee a$, whence $(e \wedge b) \vee a=(e \vee a) \wedge b$. With an obvious change in notation, $b \leqq a^{\prime} \Longrightarrow(b \vee e) \wedge a^{\prime}=b \vee\left(e \wedge a^{\prime}\right)$. Hence $M\left(e, a^{\prime}\right)$ for all $e \in L$.

Suppose conversely that $M\left(e, a^{\prime}\right)$ for all $e \in L$. In particular, if $b^{\prime} \leqq a^{\prime}$, then $\left(b^{\prime} \vee e^{\prime}\right) \wedge a^{\prime}=b^{\prime} \vee\left(e^{\prime} \wedge a^{\prime}\right)$, so that $b \geqq a \Rightarrow(b \wedge e) \vee a=$ $b \wedge(e \vee a)$. Given $f \in L, f \psi_{a} \geqq a$, so $\left(e \wedge f \psi_{a}\right) \vee a=(e \vee a) \wedge f \psi_{a}$, $\left(e \wedge f \psi_{a}\right) \psi_{a}=e \psi_{a} \wedge f \psi_{a}$. Thus $\psi_{a}$ is a weak quantifier. That $\psi_{a}$ is discrete follows from the observation that if $e \in L(a, 1)$, then $e=$ $e \vee a=e \psi_{a}$.

The only remaining problem is to consider the lattice of closed subspaces $L(H)$ of a Hilbert space $H$ and determine which elements satisfy the conditions of Theorem 13 . If $H$ is finite dimensional, $L(H)$ is modular, so $\psi_{A}$ is a weak quantifier for every $A \in L(H)$. The answer for the infinite dimensional case is contained in the next theorem.

THEOREM 14. Let $H$ be an infinite dimensional Hilbert space. Then $M(E, A)$ for all $E \in L(H)$ if and only if $A$ or $A^{\perp}$ is finite dimensional.

Proof. If $A$ is finite dimensional and $B \leqq A$, we need only show that for any $E \in L(H),(B+E) \wedge A \leqq B+(E \wedge A)$. In case $A^{\perp}$ is finite dimensional, we must show that $B \geqq A$ implies that $B \wedge\left(E+A^{\perp}\right) \leqq$ $(B \wedge E)+A^{\perp}$. Both results follow from the standard pointwise argument.

Suppose that both $A$ and $A^{\perp}$ are infinite dimensional. Using a minor variation of the construction outlined in Halmos $[4, \S 15]$, one can produce a closed subspace $N$ and a vector $y$ such that

$$
\begin{gathered}
A^{\perp}<A^{\perp}+\langle y\rangle \\
A^{\perp} \vee N^{\perp}=\left(A^{\perp}+\langle y\rangle\right) \vee N^{\perp} \\
A^{\perp} \wedge N^{\perp}=\left(A^{\perp}+\langle y\rangle\right) \wedge N^{\perp}=0 .
\end{gathered}
$$


It is then immediate that modularity fails for the pair $(N, A)$.

The main purpose of this paper was to determine all weak quantifiers on $L(H)$. Since $L(H)$ has the atomic bisection property, this has essentially been done, but for convenience we assemble the results into one final theorem.

THEOREM 15. ( i ) Every weak quantifier on $L(H)$ is discrete or indiscrete.

(ii) Given $A \in L(H)$ the mapping $B \varphi=A$ if $B \leqq A, B \varphi=1$ if $B \not A$ is an indiscrete weak quantifier; every indiscrete weak quantifier takes this form.

(iii) The mapping $B \psi_{A}=B \vee A$ is a discrete weak quantifier if and only if $A$ or $A^{\perp}$ is finite dimensional. If $\varphi$ is a discrete weak quantifier then $\varphi=\psi_{(0 \varphi)}$.

\section{BIBLIOGRAPHY}

1. D. J. Foulis, Baer*-semigroups, Proc. Amer. Math. Soc., 11, No. 4 (1960), 648-654.

2. - A note on orthomodular lattices, Port. Math., 21, Fasc. 1 (1962), 65-72.

3. P. R. Halmos, Algebraic logic I, monadic Boolean algebras, Composito Math., 12 (1955), 217-249.

4. - Introduction to Hilbert Space and the Theory of Spectral Multiplicity, Second Endition, New York (1957).

5. P. Jordan, Quantenlogik und das Kommutative Gesetz, The Axiomatic Method, with special reference to Geometry and Physics, L. Henkin, P. Suppes, A. Tarski, Amsterdam (1959), 365-375.

6. I. Kaplansky, Any orthocomplemented complete modular lattice is a continuous geometry, Ann. of Math., 61 (1955), 524-541.

7. G. W. Mackey, Quantum mechanics and Hilbert space, Herbert Ellsworth Slaught Memorial Paper No. 6, Amer. Math. Monthly, 64, No. 8 (1957), 45-57.

8. F. Maeda, Kontinuierliche Geometrien, Berlin (1958).

9. M. Nakamura, The permutability in a certain orthocomplemented lattice, Kodai Math. Sem. Rep., 9 (1957), 158-160.

10. U. Sasaki, On orthocomplemented lattices satisfying the exchange axiom, Hiroshima Japan Univ. Journ. of Sci., Ser A, 17, No. 3 (1954), 293-302.

11. J. von Neumann, Continuous Geometry, Princeton (1960). 



\section{PACIFIC JOURNAL OF MATHEMATICS}

\section{EDITORS}

RalPh S. Phillips

Stanford University

Stanford, California

M. G. Arsove

University of Washington

Seattle 5 , Washington
J. Dugundu

University of Southern California

Los Angeles 7, California

Lowell J. Paige

University of California

Los Angeles 24, California

\section{ASSOCIATE EDITORS}
E. F. BECKENBACH
D. DERRY
H. L. ROYDEN
E. G. STRAUS
T. M. CHERRY
M. OHTSUKA
E. SPANIER
F. WOLF

\section{SUPPORTING INSTITUTIONS}

\author{
UNIVERSITY OF BRITISH COLUMBIA \\ CALIFORNIA INSTITUTE OF TECHNOLOGY \\ UNIVERSITY OF CALIFORNIA \\ MONTANA STATE UNIVERSITY \\ UNIVERSITY OF NEVADA \\ NEW MEXICO STATE UNIVERSITY \\ OREGON STATE UNIVERSITY \\ UNIVERSITY OF OREGON \\ OSAKA UNIVERSITY \\ UNIVERSITY OF SOUTHERN CALIFORNIA
}

\author{
STANFORD UNIVERSITY \\ UNIVERSITY OF TOKYO \\ UNIVERSITY OF UTAH \\ WASHINGTON STATE UNIVERSITY \\ UNIVERSITY OF WASHINGTON \\ AMERICAN MATHEMATICAL SOCIETY \\ CALIFORNIA RESEARCH CORPORATION \\ SPACE TECHNOLOGY LABORATORIES \\ NAVAL ORDNANCE TEST STATION
}

Mathematical papers intended for publication in the Pacific Journal of Mathematrcs should be typewritten (double spaced), and the author should keep a complete copy. Manuscripts may be sent to any one of the four editors. All other communications to the editors should be addressed to the managing editor, L. J. Paige at the University of California, Los Angeles 24, California.

50 reprints per author of each article are furnished free of charge; additional copses may be obtained at cost in multiples of 50 .

The Pacific Journal of Mathematics is published quarterly, in March, June, September, and December. Effective with Volume 13 the price per volume (4 numbers) is $\$ 18.00$; single issues, $\$ 5.00$. Special price for current issues to individual faculty members of supporting institutions and to individual members of the American Mathematical Society: $\$ 8.00$ per volume; single issues $\$ 2.50$. Back numbers are available.

Subscriptions, orders for back numbers, and changes of address should be sent to Pacific Journal of Mathematics, 103 Highland Boulevard, Berkeley 8, California.

Printed at Kokusai Bunken Insatsusha (International Academic Printing Co., Ltd.), No. 6 , 2-chome, Fujimi-cho, Chiyoda-ku, Tokyo, Japan.

\section{PUBLISHED BY PACIFIC JOURNAL OF MATHEMATICS, A NON-PROFIT CORPORATION}

The Supporting Institutions listed above contribute to the cost of publication of this Journal, but they are not owners or publishers and have no responsibility for its content or policies. 


\section{Pacific Journal of Mathematics}

\section{Vol. 13, No. $4 \quad$ June, 1963}

Dallas O. Banks, Bounds for eigenvalues and generalized convexity ........... 1031

Jerrold William Bebernes, A subfunction approach to a boundary value problem for

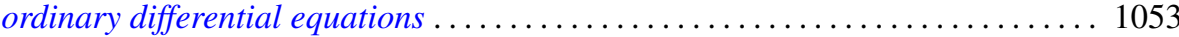

Woodrow Wilson Bledsoe and A. P. Morse, A topological measure construction . . . 1067

George Clements, Entropies of several sets of real valued functions . . . . . . . . . 1085

Sandra Barkdull Cleveland, Homomorphisms of non-commutative *-algebras . . . . . 1097

William John Andrew Culmer and William Ashton Harris, Convergent solutions of

ordinary linear homogeneous difference equations . . . . . . . . . . . . . . . 1111

Ralph DeMarr, Common fixed points for commuting contraction mappings . . . . . . 1139

James Robert Dorroh, Integral equations in normed abelian groups . . . . . . . . 1143

Adriano Mario Garsia, Entropy and singularity of infinite convolutions . . . . . . . 1159

J. J. Gergen, Francis G. Dressel and Wilbur Hallan Purcell, Jr., Convergence of extended Bernstein polynomials in the complex plane ................. 1171

Irving Leonard Glicksberg, A remark on analyticity of function algebras . . . . . . 1181

Charles John August Halberg, Jr., Semigroups of matrices defining linked operators

with different spectra ................................. 1187

Philip Hartman and Nelson Onuchic, On the asymptotic integration of ordinary

differential equations . . . . . . . . . . . . . . . . . . . . . . . . . . . . 1193

Isidore Heller, On a class of equivalent systems of linear inequalities . . . . . . . . . 1209

Joseph Hersch, The method of interior parallels applied to polygonal or multiply

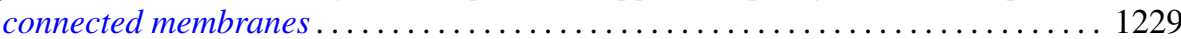

Hans F. Weinberger, An effectless cutting of a vibrating membrane . . . . . . . . . . 1239

Melvin F. Janowitz, Quantifiers and orthomodular lattices ....

Samuel Karlin and Albert Boris J. Novikoff, Generalized convex inequalities . .

Tilla Weinstein, Another conformal structure on immersed surfaces of negative

curvature.

Gregers Louis Krabbe, Spectral permanence of scalar operators

Shige Toshi Kuroda, Finite-dimensional perturbation and a representaion of

scattering operator.

Marvin David Marcus and Afton Herbert Cayford, Equality in certain

inequalities

Joseph Martin, A note on uncountably many disks .

Eugene Kay McLachlan, Extremal elements of the convex cone of semi-norms . . . . 1335

John W. Moon, An extension of Landau's theorem on tournaments . .

Louis Joel Mordell, On the integer solutions of $y(y+1)=x(x$

Kenneth Roy Mount, Some remarks on Fitting's invariants .....

Miroslav Novotný, Über Abbildungen von Mengen ............

Robert Dean Ryan, Conjugate functions in Orlicz spaces.

John Vincent Ryff, On the representation of doubly stochastic operators . . . . . . . . 1379

Donald Ray Sherbert, Banach algebras of Lipschitz functions .

James McLean Sloss, Reflection of biharmonic functions across analytic boundary

conditions with examples.

L. Bruce Treybig, Concerning homogeneity in totally ordered, connected topological space....

John Wermer, The space of real parts of a function algebra...

James Juei-Chin Yeh, Orthogonal developments of functionals and related theorems

in the Wiener space of functions of two variables......... 